Supporting Information for

\title{
Molecular Understanding of Solvents and Glycitein Interaction during Extraction
}

\author{
Hailiang Zhao ${ }^{1,2}$, Xue Song ${ }^{2}$, Yingming Zhang ${ }^{2}, \mathrm{Xia}_{\text {Sheng }}{ }^{2 *}$, and Keren $\mathrm{Gu}^{2 *}$ \\ ${ }^{1}$ Province Key Laboratory of Cereal Resource Transformation and Utilization, Henan \\ University of Technology, Lianhua Street 100, 450001 Zhengzhou, China \\ ${ }^{2}$ College of Chemistry, Chemical and Environmental Engineering, Henan University \\ of Technology, Lianhua Street 100, 450001 Zhengzhou, China \\ e-mail: xia.sheng@haut.edu.cn; gkr@haut.edu.cn
}


Table S1. Calculated binding energy (BE), Zero-point vibrational energy (ZPVE), basis set superposition error (BSSE), enthalpy of formation $\left(\Delta H_{298 K}^{\theta}\right)$, and Gibbs free energy of formation $\left(\Delta G_{298 K}^{\theta}\right)$ at $298.15 \mathrm{~K}$ and $1 \mathrm{~atm}$ for MeOH-Glycitein at the B3LYPD3/cc-pVTZ level ${ }^{\text {a }}$

\begin{tabular}{cccccc}
\hline Conformers & $B E$ & ZPVE & BSSE & $\Delta H_{298 K}^{\theta}$ & $\Delta G_{298 K}^{\theta}$ \\
\hline MeOH-Glycitein (A) & -25.1 & 5.9 & 9.9 & -34.4 & 3.9 \\
MeOH-Glycitein (B) & -17.2 & 5.0 & 7.1 & -22.7 & 12.9 \\
MeOH-Glycitein (C) & -37.2 & 6.0 & 8.1 & -44.2 & -6.4 \\
MeOH-Glycitein (D) & -13.7 & 4.6 & 6.8 & -18.6 & 16.1 \\
MeOH-Glycitein (E) & -18.0 & 5.1 & 7.2 & -23.5 & 11.8 \\
MeOH-Glycitein (F) & -29.1 & 6.6 & 7.4 & -35.5 & 2.4 \\
\hline
\end{tabular}

${ }^{\mathrm{a}}$ Energies are in $\mathrm{kJ} \mathrm{mol}^{-1} \cdot{ }^{\mathrm{b}}$ BEs are corrected with ZPVE and BSSE. 